Walfrido C. Adan, Jr., MD

Emmanuel Tadeus S. Cruz, MD

Department of Otorhinolaryngology

Head and Neck Surgery

Quezon City General Hospital
Correspondence: Dr. Emmanuel Tadeus S. Cruz Department of Otorhinolaryngology

Head and Neck Surgery

Quezon City General Hospital

Seminary Road, Barangay Bahay Toro, Project 8, QC 1106

Philippines

Phone: (632) 4261314

Email:emancrz@yahoo.com

The authors declared that this represents original material that is not being considered for publication or has not been published or accepted for publication elsewhere, in full or in part, in print or electronic media; that the manuscript has been read and approved by the authors, that the requirements for authorship have been met by the authors, and that the authors believe that the manuscript represent honest work.

Disclosures: The authors signed a disclosure that there are no financial or other (including personal) relationships, intellectual passion, political views or beliefs, and institutional affiliations that might lead to conflict of interest.

Presented at the Philippine Society of Otolaryngology Head and Neck Surgery Descriptive Research Contest. September 18, 2014. Natrapharm, The Patriot Building, Paranaque City.

\section{Association of the Laterality of Chronic Suppurative Otitis Media and Sinonasal Disease Based on Temporal Bone CT Scans and Lund Mackay Scoring System}

\begin{abstract}
Objective: To determine the association between the laterality of chronic suppurative otitis media (CSOM) and the laterality of sinonasal disease, based on temporal bone $\mathrm{CT}$ scan results and Lund-Mackay Scoring system, among patients admitted for ear surgery in a tertiary government hospital in Metro Manila.
\end{abstract}

\section{Methods:}

Design: Retrospective review of records

Setting: $\quad$ Tertiary Government Hospital

Participants: Ninety-eight (98) patients diagnosed with chronic suppurative otitis media admitted for otologic surgery in the Department of Otorhinolaryngology - Head and Neck Surgery from January 2011 to June 2014 were considered for inclusion. Hospital charts and temporal bone CT scan results were retrieved and analyzed for ear and sinonasal radiographic abnormalities and laterality. Excluded were those without CT scan plates, who underwent temporal bone surgery for reasons other than chronic suppurative otitis media, and those with incomplete records. The Lund-Mackay Scoring System was used to grade sinonasal findings which were compared to CSOM complications. Data was analyzed using t-test, ANOVA for homogenous numerical data, Kruskal-Wallis for heterogenous numerical data, and chi-square test for nominal type of data.

Results: Of the 64 patients included in the study, 12 or $18.75 \%$ had radiographic sinonasal abnormalities. There was no significant association between the laterality of ear disease and the laterality of sinonasal pathology as there was no significant difference in the proportion of subjects with sinonasal disease according to laterality of $\operatorname{CSOM}(p=.32)$. When site of nose pathology was compared to Lund-Mackay graded scores, it was found that bilateral nose pathology generally had a higher Lund-Mackay score of $8.60 \pm 5.60$. However, there was no significant difference in the Lund-Mackay score according to the nose pathology site $(p=.20)$. An association was seen between total LMS and patients with ear pathologies, but no significant difference was noted $(p=.44)$. Although patients with ear complications had higher LM scores, this was not statistically significant.

Conclusion: Laterality of ear disease was not associated with the laterality of sinonasal disease, although CSOM complications were associated with high Lund-Mackay scores. Future, betterdesigned studies may shed more light on these associations.

Keywords: chronic suppurative otitis media, sinonasal disease, Lund-Mackay, Temporal Bone CT scan 
Patients suffering from chronic suppurative otitis media (CSOM) may have underlying sinonasal problems. In children, otitis media is usually preceded by upper respiratory tract infections. This may be related to the wider, shorter, and more horizontally-oriented eustachian tube in children.'

Yoelekar and Dasgupta related the origin of chronic suppurative otitis media to sinonasal pathology, particularly eustachian tube dysfunction. ${ }^{2} \quad$ Out of the 200 patients in the study, 50\% had tubotympanic type chronic suppurative otitis media, $80 \%$ had deviated nasal septum, $13.5 \%$ sinusitis, $10.5 \%$ nasal polyps. ${ }^{2}$ However, diagnosis was based on physical examination and endoscopy and use of imaging was not mentioned. Neither was an association between the laterality of ear disease and laterality of paranasal sinus disease determined. A PubMed review of MEDLINE using the keywords "sinonasal disease," "chronic suppurative otitis media," and "laterality" yielded a paucity of data on the relationship between chronic suppurative otitis media and laterality of sinonasal disease.

Because the paranasal sinuses can be visualized on routine temporal bone CT scans in our institution, we aimed to determine the association between laterality of chronic suppurative otitis media and laterality of sinonasal disease among CSOM patients admitted for surgery based on temporal bone $\mathrm{CT}$ scan results.

\section{METHODS}

This retrospective review of records was approved by the Quezon City General Hospital Research Review Board. Ninety-eight (98) patients diagnosed with chronic suppurative otitis media, admitted for ear surgery under the Department of Otorhinolaryngology - Head and Neck Surgery of the Quezon City General Hospital from January 2011 to June 2014 were initially considered for inclusion. Hospital records and plain temporal bone $\mathrm{CT}$ scans were retrieved. Excluded were those without CT scan plates, who underwent temporal bone surgery for reasons other than chronic suppurative otitis media, and those with incomplete records.

Using Epi Info Version 7 (Centers for Disease Control, Atlanta, GA, USA) software, the minimum sample size was estimated to be at least 61 using the following parameters: Alpha $(a)=5 \%$, with the prevalence of sinonasal disease in the Philippines at $20 \%{ }^{4}$ with a maximum tolerable error $(\mathrm{e})=10$. Data were encoded and tallied in SPSS version 10 for windows (Statistical Package for the Social Sciences Inc., Chicago, IL, USA).

Patient records were analyzed and anonymized data on the history (duration of ear discharge, presence of chronic suppurative otitis media complications and nasal symptoms) and physical examination (otoscopy, tuning fork test, rhinoscopy and nasal endoscopy) with emphasis on the ear and sinonasal details were tabulated.

All the temporal bone $\mathrm{CT}$ scan results were evaluated by a single board-certified radiologist for the presence of cholesteatoma, soft tissue densities and bony defects. To describe the laterality of the diseased ear, the following numbers were designated: 1-Left, 2-Right, 3-Bilateral.

The same radiologist was oriented to, and graded the severity of sinonasal disease using the Lund-Mackay Scoring System. ${ }^{3}$ The laterality of sinonasal disease was designated: 0-none, 1-Left, 2-Right, 3-Bilateral. Based on the grading system, the right or left sinuses were divided into 6 portions: maxillary, anterior and posterior ethmoid, sphenoid, frontal sinuses, and ostiomeatal complex. The severity of sinus mucosal inflammation or fluid accumulation was scored as follows: 0 for complete lucency, 1 for partial lucency and 2 for complete opacity. In addition, the ostiomeatal complex was scored as either 0 for not obstructed or 2 for obstructed. The 10 scores for the various sinuses and bilateral ostiomeatal complexes were summed up to give a bilateral total Lund-Mackay Score that could range from 0 (complete lucency of all sinuses) to 24 (complete opacity of all sinuses). ${ }^{3} \mathrm{~A}$ bilateral score of 5 and above, and a unilateral score of 4 and above was considered severe sinonasal disease.

The association between the laterality of chronic suppurative otitis media and laterality of sinonasal disease was determined and the unilateral and bilateral Lund-MacKay scores were compared against ears with, and without complications. Descriptive statistics were generated for all variables. For nominal data frequencies and percentages were computed. For numerical data, mean \pm SD were generated.

Medians were computed for non-homogenous data, specifically in the comparison of Lund-Mackay Scoring of subjects with or without ear complications. The one-way analysis of variance (ANOVA) was used for comparing the Lund-Mackay Scoring according to nose or ear pathology site. However, if there was a difference in the homogeneity of the data the non-parametric equivalent of the ANOVA, the Kruskal Wallis test-- was used instead. Chi-square test was used for determining the association between laterality of CSOM and sinonasal disease. T-Test was used in comparing the LMS Score with the presence of complications of CSOM. Level of significance was set at $p<.05$.

\section{RESULTS}

A total of 64 patients were finally included in the study, 37 (57.8\%) female and $27(42.2 \%)$ male. The mean age was 37 years old (range 4 - 77 years old). Majority (54) were service patients belonging to lower socio-economic brackets while the remaining 10 were private patients.

All 64 patients underwent ear surgery, 25 (39\%) had intact canal wall mastoidectomy while $39(61 \%)$ had canal wall down mastoidectomy. Thirty-four (34) right and 31 left ears were operated on with a total of 36 tympanoplasties performed. Of the 64 mastoidectomies, 30 (46.88\%) had complications of chronic suppurative otitis media such as cholesteatoma (23), brain abscess (6), subperiosteal abscess (3), suppurative labyrinthitis (2), Bezold Abscess (2), Gradenigo Syndrome (2), facial nerve paralysis (1), aural polyp (1). Hearing loss was seen in 
varying severity among all patients who had a pure tone audiometry done. On review of the temporal bone CT scans, 17 patients had radiographic findings of cholesteatoma solely in the left ear, 18 patients in the right ear, and 30 patients had bilateral ear disease.

With regard to the laterality of ear disease, the distribution was almost equal between right and left ears with 15/64 and 18/64 patients, respectively. However, there were more cases (31/64) with bilateral ear disease than unilateral right or left ear abnormality.

With regard to the sinonasal findings, out of the 64 subjects, only 12 (18.75\%) had positive findings on CT scan, while the other 52 patients had normal findings. Of the 12 patients, 7 (58.3\%) had bilateral involvement, only 1 (8.33\%) had left-sided sinonasal pathology and 4 (33.3\%) had right-sided sinonasal involvement. Using the chi-square test, there was no significant difference in the proportion of subjects with sinonasal disease according to laterality of otitis media $(p=.32)$. (Table 1)

Table 1. Distribution of Subjects with Sinonasal Disease According to Laterality of Chronic Suppurative Otitis Media Patients

\begin{tabular}{|l|c|c|c|}
\multirow{2}{*}{\multicolumn{1}{|c|}{ CSOM }} & \multicolumn{2}{|c|}{ Sinonasal Disease } & \multirow{2}{*}{ Total } \\
\cline { 2 - 3 } & $\begin{array}{c}(\boldsymbol{+}) \\
(\mathbf{n} / \mathbf{1 2}), \%\end{array}$ & $\begin{array}{c}(-) \\
(\mathbf{n} / \mathbf{5 2}), \%\end{array}$ & \\
\hline Left Ear & $1(8.3)$ & $15(28.8)$ & 16 \\
Right Ear & $4(33.3)$ & $15(28.8)$ & 19 \\
Bilateral & $7(58.3)$ & $22(42.3)$ & 29 \\
Total & 12 & 52 & 64 \\
\hline
\end{tabular}

$P=.32$ (Not Significant)

Table 2 shows the association of the laterality of CSOM with the laterality of sinonasal disease among the 12 patients who had sinonasal finding on temporal bone CT scan. Only one patient had left ear pathology with bilateral nose pathology. Of the 4 patients with right ear pathology, 1 had left nose pathology and 3 had bilateral nose pathology, none had right nose pathology. Seven had bilateral otitis media in whom 1 had right nose pathology and 6 had bilateral nose pathology. Analysis by chi-square test showed that there was no significant association between laterality of CSOM with the laterality of sinonasal disease $(p=.59)$

The 12 patients with sinonasal pathologies on CT scan were graded using the Lund-Mackay scoring system. The average Lund-Mackay score was 7, with the highest score of 17 and the lowest of 2.

Table 2. Association of the Laterality of CSOM with Laterality of Sinonasal Disease

\begin{tabular}{|l|l|l|l|c}
\multirow{2}{*}{ CSOM } & \multicolumn{3}{|c|}{ Sinonasal Disease } & \multirow{2}{*}{ Total } \\
\cline { 2 - 4 } & Left & Right & Bilateral & \\
\hline Left Ear & 0 & 0 & $1(1.6 \%)$ & 1 \\
Right Ear & $1(1.6 \%)$ & 0 & $3(4.7 \%)$ & 4 \\
Bilateral & 0 & $1(1.6 \%)$ & $6(9.4 \%)$ & 7 \\
Total & 1 & 1 & 10 & 12 \\
\hline
\end{tabular}

$p=.59$ (Not Significant)
When the site of nose pathology was compared to the Lund-Mackay graded score, heterogeneity in the data set was found, thus a KruskalWallis test was used. This analysis showed that bilateral nose pathology generally had a higher Lund-Mackay score of $8.60 \pm 5.60$, compared to no pathology at $0.10 \pm 0.50$, left nose pathology at $3.00 \pm 0$, and right nose pathology at $2.00 \pm 0.00$. However, there was no significant difference in the Lund-Mackay score according to the nose pathology site $(p=.20)$.

Table 3. Lund-Mackay Scoring

\begin{tabular}{|l|c|c|c|}
\hline $\begin{array}{c}\text { Nose Pathology } \\
\text { Site }\end{array}$ & $\begin{array}{c}\text { Frequency } \\
(\mathbf{n}=64)\end{array}$ & $\begin{array}{c}\text { Mean } \pm \text { SD } \\
\text { (Median) }\end{array}$ & *p-value \\
\hline None & 52 & $0.01 \pm 0.50$ & $<.01(S)^{\dagger}$ \\
Left & 1 & $3.00 \pm 0.00(3)$ & - \\
Right & 1 & $2.00 \pm 0.00(2)$ & - \\
Bilateral & 10 & $8.60 \pm 5.60(7)$ & $<.01(S)^{\ddagger}$ \\
\hline
\end{tabular}

* $p>$.05- Not significant; $p \leq$.05-Significant

$t$-between none and not none (with)

$\neq$ - between bilateral and none only

Comparison of the Lund-Mackay to ear pathology site using ANOVA yielded no statistical significance $(p=.44)$, although the left ear had a higher score compared to right and bilateral ear pathologies. (Table 4)

Table 4. Lund-Mackay Scoring of Patients with Sinonasal Disease According to Ear Pathology Site

\begin{tabular}{|l|c|c|c|}
\hline & $\begin{array}{c}\text { Frequency } \\
\text { (n/12) }\end{array}$ & $\begin{array}{c}\text { Mean } \pm \text { SD } \\
\text { (Median) }\end{array}$ & *p-value \\
\hline Ear Pathology Site & 1 & $14.00 \pm 0.00(14)$ & $1.00(\mathrm{NS})^{\dagger}$ \\
Left & 4 & $9.25 \pm 7.32(8.5)$ & $1.00(\mathrm{NS})^{\ddagger}$ \\
Right & 7 & $5.71 \pm 4.23(4)$ & $.23(\mathrm{NS})^{\S}$ \\
Bilateral & 12 & & \\
Total & &
\end{tabular}

${ }^{*} p>$.05- Not significant;

$t$ - between none and not none

F- between bilateral and none only

$\S$ - between bilateral and not bilateral

Of the 12 patients with positive sinonasal findings, 7 (58.3\%) had ear complications such as cholesteatoma, subperiosteal abscess and brain abscess. It was observed that these patients with ear complications generally obtained higher Lund-Mackay scores. The ear complication patients had an average Lund-Mackay score of 9.28, compared to the score of patients without ear complications with a 5.20 Lund-Mackay score average. T-test analysis showed that the difference was not statistically significant $(p=.22)$. (Table 5)

Table 5. Lund-Mackay Scoring of Patients with Sinonasal Disease According to Ear Complications

\begin{tabular}{|c|c|c|c|}
\hline $\begin{array}{c}\text { Ear } \\
\text { Complications }\end{array}$ & $\begin{array}{c}\text { Frequency } \\
(\mathbf{n = 1 2 )}\end{array}$ & Mean \pm SD & *p-value \\
\hline With & 7 & $9.28 \pm 5.74$ & $.22(\mathrm{NS})$ \\
Without & 5 & $5.20 \pm 4.97$ & $.22(\mathrm{NS})$ \\
\hline
\end{tabular}

${ }^{*} p>$.05-Not significant; $p \leq .05$-Significant 


\section{DISCUSSION}

There was no association between laterality of chronic ear disease and sinonasal disease in this study. This was supported by a low $18.75 \%$ incidence of sinonasal abnormalities in the sample population of surgical ear patients, where only 12 patients out of 64 with CSOM had positive findings of sinonasal disease on CT scan.

In spite of the reasonable hypothesis that a unilateral ear problem should be associated with ipsilateral sinonasal problems, we found no such correlation between the laterality of sinonasal disease and CSOM. While this may be due to our failure to differentiate between atticoantral and tubotympanic otitis media, as well as failure to catch previous sinonasal disease that may have precipitated ear disease but was subsequently treated, or sinonasal disease that was not severe enough to manifest radiographically, it may still be argued that in patients with CSOM a thorough examination of all the sinuses, and not just the ipsilateral sinuses, should be performed. Having said that, by focusing on radiographic findings, we failed to analyze the histories and physical examination findings of otitis and sinonasal disease in our sample; additional data that may have strengthened the association of laterality. Despite these shortcomings, the correlation seen between complications of CSOM and severity of sinonasal disease support the association between ear and sinonasal problems.

A study by Yeolekar and Dasgupta relied on history and anterior rhinoscopy to diagnose sinonasal disease ${ }^{2}$ without the benefit of a CT scan which may show evidence of radiographic abnormalities in the paranasal sinuses. Because the paranasal sinuses and ostiomeatal complexes can be visualized in routine temporal bone CT scans in our institution, this study intended to go further by investigating radiographic evidence of sinonasal disease among CSOM patients admitted for surgery. Unfortunately, we erred on the opposite side of Yeolekar and Dasgupta's study ${ }^{2}$ by focusing on imaging to the detriment of history and anterior rhinoscopy.

A study by Chen et al. in Taiwan, using head and neck CT scans in 600 patients determined a control dataset for Taiwanese individuals to show what bilateral and unilateral right and left LMS score was considered severe. ${ }^{3}$ Bilateral total LMS was separated from unilaterally total LMS because there were significant differences found among bilateral, control, unilateral right and unilateral left sinuses groups. After exclusions, there were 490 study subjects and 119 controls. In the Taiwan study group, the bilaterally total LMS obtained for the dataset was $0.96 \pm 1.91$ (mean \pm SD) with a right total LMS of $0.46 \pm 1.28$ and a left total LMS of $0.50 \pm 1.41$. Thus, a patient with bilateral total LMS of more than 5 (considered severe), or with a unilateral LMS of more than 4 , was considered beyond $97.7 \%$ of the common population. ${ }^{3}$ These thresholds were used in this study to indicate the severity of our findings. When we compared the site of nose pathology to the LMS in this study, no significant difference was found $(p=0.20)$. This is in contrast with the Taiwan study, wherein they had to separate the right from left and bilateral pathology since significant differences were found in their data set. Since no significant difference was found in our data set, the LMS was treated as a total score and not individually compared against each other.

In this study, 6 or $9.38 \%$ of the sample population had a bilateral total LMS score greater than 5 . The association between high LundMackay scores, which reflect the severity of sinonasal disease, and CSOM complications among patients in this study, warrants further inquiry. It may also be interesting to note that in our dataset, the left ear $(14.00 \pm 0.00 \mathrm{SD}=14)$ had a higher score compared to the right or bilateral ear pathology.

A major limitation of this study was the non-segregation of atticoantral from tubo-tympanic CSOM. The former would theoretically have little or no relation to sinonasal disease, which the latter would be expected to have. Our definition of CSOM complications (which strictly speaking, include tympanic membrane perforation and hearing impairment) is another limitation of our paper. We only used the term to apply to more serious complications listed in our results (various abscesses, suppurative labyrinthitis, Gradenigo syndrome, facial nerve paralysis), and yet, we also included cholesteatoma and aural polyp. Despite our retrieving history and physical examination results, we did not consider a history of hearing impairment and hearing test results (which were also incomplete), or tympanic membrane perforations in our list of complications. In addition, co-morbidities identified among our patients were not analyzed against ear and sinonasal complications and actual physical examination findings. Other limitations include the unvaliditated use of temporal bone CT scans in interpreting and grading the severity of sinonasal disease using the Lund-Mackay Scoring System.

Although laterality of ear disease was not associated with the laterality of sinonasal disease in this study, CSOM complications were associated with high Lund-Mackay scores. Future, more rigorouslydesigned studies with a larger and more diverse population may yet determine an association between CSOM and laterality of sinonasal disease. A better-designed study with a larger population may also establish the predictive value of a high LMS for CSOM complications.

\section{REFERENCES}

1. Acuin J. Chronic Suppurative Otitis Media Burden of Illness and Management Options. Child and Adolescent Health and Development Prevention of Blindness and Deafness. World Health Organization. Geneva, Switzerland. 2004; p.9-15.

2. Yeolekar AM, Dasgupta KS. Otitis media: Does the onus lie on sinonasal pathology? Indian J Otol [serial online] 2011 Oct;17(1):8-11. [cited 2014 Jul 10]. Available from: http://www.indianjotol. org/text.asp?2011/17/1/8/85784 DOI: 10.4103/0971-7749.85784

3. Chen J-J, Chen D-L, Chen C-J. The Lund-Mackay Score for Adult Head and Neck Computed Tomography. J Radio/ Sci. 2011; 36(4): 203-208. [cited 2014 Aug 1]; Available from: http://www. rsroc.org.tw/db/Jrs/article/N36/N4/360402.pdf.

4. Abong JM, Kwong SL, Alava HD, Castor MA, De Leon, JC. Prevalence of Allergic Rhinitis in Filipino Adults Based on the National Nutrition and Health Survey 2008. Asia Pac Allergy 2012 Apr;2(2):129-135. [cited 2013 March 19]. Available from: http://dx.doi.org/10.5415/ apallergy.2012.2.2.129

5 Leung RS, Katial R. The diagnosis and management of acute and chronic sinusitis. Prim Care 2008 Mar; 35(1): 11-24. DOI: 10.1016/j.pop.2007.09.002 PMID: 18206715. 